\title{
Fenología de Schizolobium PaRAHyba y VOCHYSIA GUATEMALENSIS EN UNA SELVA ALTA PERENNIFOLIA de OAxaca, México
}

\author{
Elizandro Pineda-Herrera', Juan Ignacio Valdez-Hernández y Miguel Ángel López-López \\ Programa Forestal, Colegio de Postgraduados, Montecillo, Estado de México, México \\ ${ }^{1}$ Autor para la correspondencia: elherrera2001@yahoo.com.mx. Dirección actual: doctorado en \\ Ciencias Biológicas y de la Salud, Universidad Autónoma Metropolitana Unidad Iztapalapa, México, D.F.
}

\begin{abstract}
Resumen: Se evaluaron cuatro eventos fenológicos (floración, fructificación, caída y brote de hojas) en Schizolobium parahyba (Vell.) Blake y Vochysia guatemalensis Donn.Smith., dos especies arbóreas de la selva húmeda en el norte de Oaxaca, México, con relación a la precipitación y temperatura del aire durante un año (septiembre 2006-septiembre 2007). Además, la humedad del suelo y el potencial hídrico de los árboles se midió al final de la sequía (abril-mayo 2007) e inicio de las lluvias (junio-julio 2007). Los resultados mostraron correlaciones significativas $(P<0.01)$ entre la precipitación y la fructificación en ambas especies $(r=$ $0.89)$ y el brote de hojas en $S$. parahyba $(r=0.88)$. Los valores registrados de humedad del suelo (\%) y potencial hídrico (bar) fueron: $S$. parahyba (8 a 25\%, -11 a -1 bar) y $V$. guatemalensis (13 a 30\%, -5.8 a -1.5 bar). El estrés hídrico fue mayor en algunos árboles de $S$. parahyba que en todos los de V. guatemalensis. Schizolobium parahyba es una especie cuya fenología está asociada a la estacionalidad del clima y V. guatemalensis no tiene una fenología determinada por la estacionalidad climática, sino por factores endógenos o geográficos.
\end{abstract}

Palabras clave: brote de hojas, caída de hojas, estacionalidad climática, floración, fructificación.

\begin{abstract}
Four phenological events (flowering, fruiting, leaf fall, and leaf flushing) were evaluated in Schizolobium parahyba (Vell.) Blake and Vochysia guatemalensis Donn.Smith., two tree species of the humid tropical forest in the north of Oaxaca, México, to relate with rainfall and air temperature during a year (September 2006-September 2007). In addition, soil moisture and tree water potential were measured by the end of drought (April-May 2007) and beginning of rainy season (June-July 2007). Results show that rainfall was significantly $(P<0.01)$ correlated with fruiting in both species $(r=0.89)$ and with leaf flush in $S$. parahyba $(r=0.88)$. The values of soil moisture (\%) and tree water potential (bars) were: $S$. parahyba (8 to $25 \%,-11$ to -1 bar) and $V$. guatemalensis (13 to 30\%, -5.8 to -1.5 bar). The water stress was higher in some trees of S. parahyba than in all trees of V. guatemalensis. The phenology of S. parahyba was associated with climatic seasonality. The phenology of V. guatemalensis maybe determined by geographic and endogenous variables.
\end{abstract}

Key words: climatic seasonality, flowering, fruiting, leaf fall, leaf flushing.

L os eventos fenológicos en los vegetales son resultado de interacciones con factores de carácter biótico como los dispersores y los polinizadores (Borchert, 1983), además de fluctuaciones de factores abióticos como el clima (Van Schaick et al., 1993). En las plantas tropicales la variabilidad de eventos fenológicos se atribuye a la estacionalidad de la precipitación (Borchert, 1999; Baker et al., 2002) y la temperatura (Gómez-Figueroa y Fournier 1995; Urrego y del Valle, 2001), la radiación solar (Wright y Van Schaick, 1994), la latitud o la altitud (Van Schaick et al., 1993).
En selvas perennifolias del mundo con precipitaciones anuales de 3,950 mm en Douala, Camerún (Walter, 1977), 4,000 mm en Los Tuxtlas, México (Soto y Gama, 1997), $3,000 \mathrm{~mm}$ en Malasia (Sakai et al., 2006) y 2,900 mm en Suva, Nueva Guinea (Walter, 1977), se ha estimado que no hay periodos de sequía marcados, el mismo comportamiento se atribuye a fluctuaciones en la temperatura. Se ha reportado que una estacionalidad poco marcada no determina la fenología en selvas perennifolias de México (Ochoa et al., 2008), Costa Rica (Vilchez et al., 2004), Panamá (Wright, 
1991), Colombia (Urrego y Del Valle, 2001) y Brasil (Morellato et al., 2000) para ningún evento. Tampoco se encontraron coincidencias en India (Bath, 1992), en Filipinas se ha demostrado que no hay participación directa con las fenofases (Hamman, 2004) y en Malasia se ha sugerido para varias especies que sus fenofases no son estacionales (Lambert y Marshall, 1991), atribuyendo los cambios a factores endógenos (genética y fisiología) o geográficos (latitud y altitud (Van Schaick et al., 1993).

No obstante, hay abundante evidencia sobre la estacionalidad de la fenología en regiones tropicales húmedas, Brenes-Cambronero y D'Stefano (2002) mencionan que hay correlación positiva de la temperatura con la caída de hojas, Tutin y Fernández (1993) sugieren una relación entre este mismo elemento y la fructificación; Haugaasen y Peres (2005) registraron la relación negativa de temperaturas mínimas con producción, tamaño y maduración de los frutos. También se reportan correlaciones significativas negativas entre la precipitación con la floración y positivas con la fructificación en Norte (Carabias y Guevara, 1985; Ibarra et al., 1991), Centro (O’Brien et al., 2008; Solis et al., 2009) y Sudamérica (Bendix et al., 2006; Zarate et al., 2006), África (Bollen y Donati, 2005) y Asia (Van Shaick, 1986).

Tomando en cuenta las discrepancias entre selvas perennifolias respecto a la estacionalidad del clima y su correlación con la fenología, es posible que para muchas especies y regiones se desconozcan estos procesos; además del papel que tienen los periodos de menor precipitación en las diferentes fenofases. Chairul et al. (2010) consideran que la relación sequía-fenología no se ha estudiado lo suficiente en este tipo de selvas, siendo un tópico de interés en ecología para entender la dinámica de poblaciones y comunidades (Wright y Cornejo, 1990); además de contribuir a rehabilitar ecosistemas con ayuda de valores como el potencial hídrico, la capacidad de campo (CC), el punto de marchitamiento permanente (PMP) y la humedad disponible del suelo (Díaz, 2001).

En el norte de Oaxaca, México, se encuentra la tercera zona con mayor extensión de selva alta perennifolia del país (Miranda y Hernández, 1963; De Teresa, 1991), ahí crecen especies arbóreas ecológica y silvícolamente importantes como Schizolobium parahyba (Vell.) Blake y Vochysia guatemalensis Donn.Smith., ambas pioneras en la sucesión secundaria e importantes para rehabilitar áreas perturbadas por ganadería y agricultura itinerante. El conocimiento de su fenología y relación con los factores abióticos y bióticos es importante para comprender su autoecología, así como promover su conservación y aprovechamiento (Bawa et al., 1989; Hartshorn, 1995). El presente trabajo tuvo como objetivos evaluar en $S$. parahyba y $V$. guatemalensis cuatro fenofases (floración, fructificación, caída y brote de hojas) y correlacionarlas con la precipitación y temperatura del aire (para un año), así como analizar la humedad del suelo y el potencial hídrico de una muestra de árboles durante el periodo final de la sequía-inicio de lluvias. Las preguntas de investigación fueron: a. si las selvas altas perennifolias no presentan fluctuaciones en su clima, S. parahyba y $V$. guatemalensis en el norte de Oaxaca no presentarán un patrón estacional en su fenología y b. durante el periodo de menor precipitación en las selvas altas del norte de Oaxaca, la disminución de la humedad del suelo y de los valores de potencial hídrico de ambas especies participan en la estacionalidad de sus eventos fenológicos.

\section{Materiales y métodos}

Área de estudio. Se encuentra en la comunidad de San Martín Soyolapan, localizada en la cuenca del Río Papaloapan, en las estribaciones de la Sierra Norte de Oaxaca; políticamente pertenece al municipio de Santiago Comaltepec, el cual a su vez forma parte del Distrito de Ixtlán de Juárez. Se ubica en las coordenadas $17^{\circ} 41^{\prime} \mathrm{N}, 96^{\circ} 16^{\prime} \mathrm{O}$, a una altitud de 60 m s.n.m. El clima es de tipo Am e(g)w": cálido húmedo con lluvias en verano, precipitación promedio anual de 3,590 $\mathrm{mm}$ y temperatura media anual de $24{ }^{\circ} \mathrm{C}$ (García, 1987). Geológicamente se encuentran afloramientos de rocas ígneas extrusivas y calizas, con los siguientes grupos de suelos: rojo-amarillentos lateríticos, amarillo-rojizos latosólicos, litosuelos kársticos, migajones rojos y lateritas (INEGI, 2011).

La vegetación predominante corresponde a selva alta perennifolia con abundancia de las siguientes especies arbóreas: Andira galeotiana, Brosimum alicastrum, Dialium guianense, Terminalia amazonia y Vochysia guatemalensis (Miranda y Hernández, 1963).

Especies de estudio. Schizolobium parahyba es nombrado comúnmente como: Picho, Palo de Picho, Brazilian fern tree, false tree-fern, guapiruvu, guapuruvu, se distribuye desde México hasta Brasil. Son árboles erectos con una copa abierta, altura de hasta $40 \mathrm{~m}$, follaje escaso, se regenera y desarrolla bien en áreas abiertas por lo que puede ser apropiada para restauración de la cobertura vegetal, ha sido empleada en construcciones rurales ligeras, obtención de celulosa y en sistemas agroforestales (Rosales et al., 1999).

Vochysia guatemalensis comúnmente se le nombra: Chancho blanco, Mayo, Barbachele, Palo de agua, San Juan, Cebo, Maca, se distribuye desde México hasta Panamá. Son árboles de 15-40 m de alto, tronco cilíndrico, frecuente observarlo creciendo en los márgenes de ríos, suelos anegados o suelo aluvional, pero además en terrenos con buen drenaje. Se le ha empleado en construcciones rurales, fabricación de muebles y embalajes (COSEFORMA, 2001).

Selección de árboles. Se eligieron 20 individuos en etapa reproductiva, diez de Schizolobium parahyba y diez de Vochysia guatemalensis, numerándose según su aparición en el campo (Fournier y Charpantier, 1975; García y Di Stefano, 2005). Los veinte árboles estuvieron repartidos homogéneamente en cuatro sitios: para $S$. parahyba sitio 1 "Pichos" 
Fenología de Schizolobium parahyba y Vochysia guatemalensis en una selva alta Perennifolia de OAXaca, México

Cuadro 1 Medición de variables biofísicas en el área de estudio

\begin{tabular}{|c|c|c|c|c|c|}
\hline Variable & $\begin{array}{l}\text { Número de } \\
\text { árboles muestreados } \\
\text { por especie }\end{array}$ & $\begin{array}{c}\text { Fechas de } \\
\text { muestreo }\end{array}$ & Lugar del muestreo & $\begin{array}{l}\text { Número de } \\
\text { muestras }\end{array}$ & Método \\
\hline $\begin{array}{l}\text { Potencial } \\
\text { hídrico }\end{array}$ & Seis & $\begin{array}{l}\text { abril, mayo, junio, } \\
\text { julio de } 2007 \\
\text { (fines de mes) }\end{array}$ & $\begin{array}{l}\text { Distintas ramas } \\
\text { de cada árbol }\end{array}$ & Tres & $\begin{array}{c}\text { Bomba de Scholander } \\
\text { (Soil Moisture, USA) } \\
\text { (Ritchie y Hinckley, 1975) }\end{array}$ \\
\hline $\begin{array}{c}\text { Humedad } \\
\text { del suelo }\end{array}$ & Dos & $\begin{array}{l}\text { abril, mayo, junio, } \\
\text { julio de } 2007 \\
\text { (fines de mes) }\end{array}$ & $\begin{array}{l}\text {-A } 1 \text { m de la base } \\
\text { del árbol (dos muestras a } \\
20 \mathrm{~cm} \text { de profundidad) } \\
\text {-En borde de proyección } \\
\text { de la copa (dos muestras a } \\
20 \mathrm{~cm} \text { de profundidad) }\end{array}$ & Cuatro & $\begin{array}{c}\text { Extractivo: Barrena } \\
\text { edafológica, } \\
\text { Analítico: } \\
\text { Gravimétrico } \\
\text { (Aguilera, 1989) }\end{array}$ \\
\hline
\end{tabular}

(sin perturbación durante seis años) y sitio 2 "Potrero" (uso ganadero reciente), para V. guatemalensis sitio 3 "La Curva" (sin perturbación durante 20 años) y sitio 4 "Cafetal" (uso agrícola pasado, abandonado hace diez años).

Registro de fenofases. Bimestralmente (septiembre 2006septiembre 2007) se llevaron a cabo observaciones mediante el uso de binoculares registrando: floración, fructificación, caída y brote de hojas, expresados en una escala del 0 al 4 según el porcentaje en que apareció cada fenofase. La escala para el registro de fenofases fue 0 (ausencia total del evento), 1 (1 a $25 \%), 2$ (26 a 50\%), 3 (51 a $75 \%) 4$ (76 a 100\%) (Fournier, 1974; García y Di Stefano, 2005).

Análisis de datos. Se elaboraron dendrofenogramas sensu Fournier, 1976) para cada especie y se calcularon coeficientes de correlación paramétrica de Pearson (Marques, 2004) para los valores promedio de cada fenofase con la precipitación temperatura del aire (promedio, mínima, máxima) usando el paquete estadístico STATA. Los datos de precipitación y temperatura fueron obtenidos del Servicio Meteorológico Nacional (SMN, 2007).

Potencial hídrico y humedad del suelo. Se midió el potencial hídrico en las primeras horas del día (6 a 7 a.m.) con el propósito de obtener datos de los árboles sin transpiración y se obtuvieron muestras de suelo de $100 \mathrm{ml}$ para determinar su humedad relativa (Cuadro 1).

En junio de 2007 por única ocasión se tomó una muestra de $500 \mathrm{ml}$ de suelo en el borde de proyección de copa de cuatro árboles en observación (uno por cada sitio) a una profundidad de $30 \mathrm{~cm}$. Con estas cuatro muestras se elaboraron curvas de retención de humedad en el laboratorio de física de suelos del Colegio de Postgraduados, las cuales sirvieron para determinar el punto de marchitamiento permanente(PMP), capacidad de campo (CC) y humedad disponible en el suelo (Aguilera, 1989).

Cuadro 2. Valores promedio de fenofases para Schizolobium parahyba y Vochysia guatemalensis en San Martín Soyolapan, Oaxaca.

\begin{tabular}{|c|c|c|c|c|c|c|c|c|}
\hline \multicolumn{5}{|c|}{ Schizolobium parahyba } & \multicolumn{4}{|c|}{ Vochysia guatemalensis } \\
\hline \multirow[b]{2}{*}{ Mes } & \multirow[b]{2}{*}{ Floración } & \multirow[b]{2}{*}{ Fructificación } & \multicolumn{2}{|c|}{ Hojas } & \multirow[b]{2}{*}{ Floración } & \multirow[b]{2}{*}{ Fructificación } & \multicolumn{2}{|c|}{ Hojas } \\
\hline & & & Caída & Brotación & & & Caída & Brotación \\
\hline $\begin{array}{c}\text { septiembre } \\
2006\end{array}$ & 0 & 3 & 0 & 3 & 0 & 3 & 0 & 2 \\
\hline $\begin{array}{c}\text { noviembre } \\
2006\end{array}$ & 0 & 0 & 0 & 2 & 0 & 0 & 0 & 2.5 \\
\hline $\begin{array}{l}\text { enero } \\
2007\end{array}$ & 0 & 0 & 0 & 2 & 0 & 0 & 0 & 1.1 \\
\hline $\begin{array}{c}\text { marzo } \\
2007\end{array}$ & 3.2 & 0 & 2.5 & 0.6 & 0 & 0 & 1.9 & 1.8 \\
\hline $\begin{array}{l}\text { mayo } \\
2007\end{array}$ & 3.8 & 0 & 3 & 1 & 3 & 0 & 2.7 & 2 \\
\hline $\begin{array}{l}\text { julio } \\
2007\end{array}$ & 0 & 1.6 & 0 & 2.5 & 3.4 & 3 & 0.7 & 2 \\
\hline $\begin{array}{c}\text { septiembre } \\
2007\end{array}$ & 0 & 2 & 0 & 2.5 & 1 & 3 & 0 & 2 \\
\hline
\end{tabular}




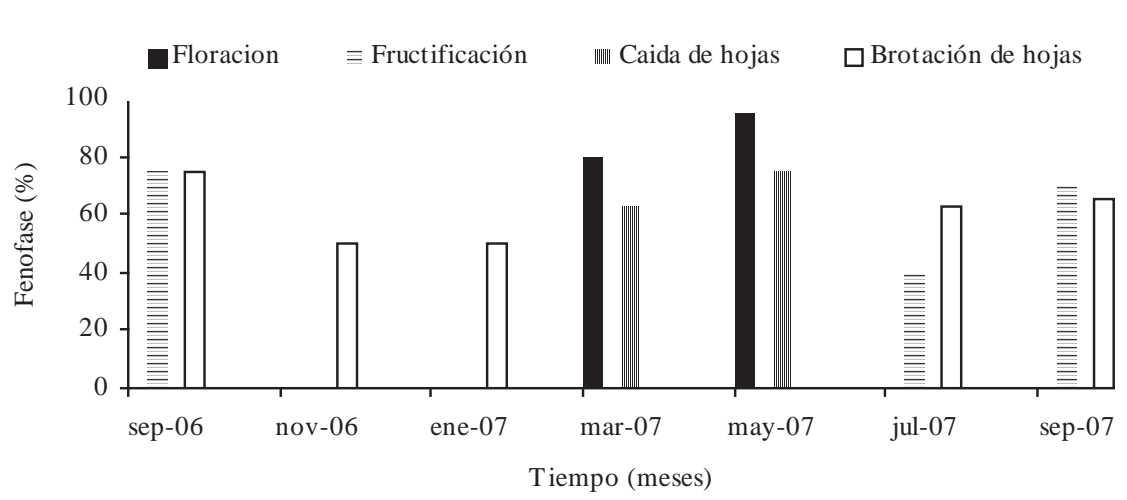

A
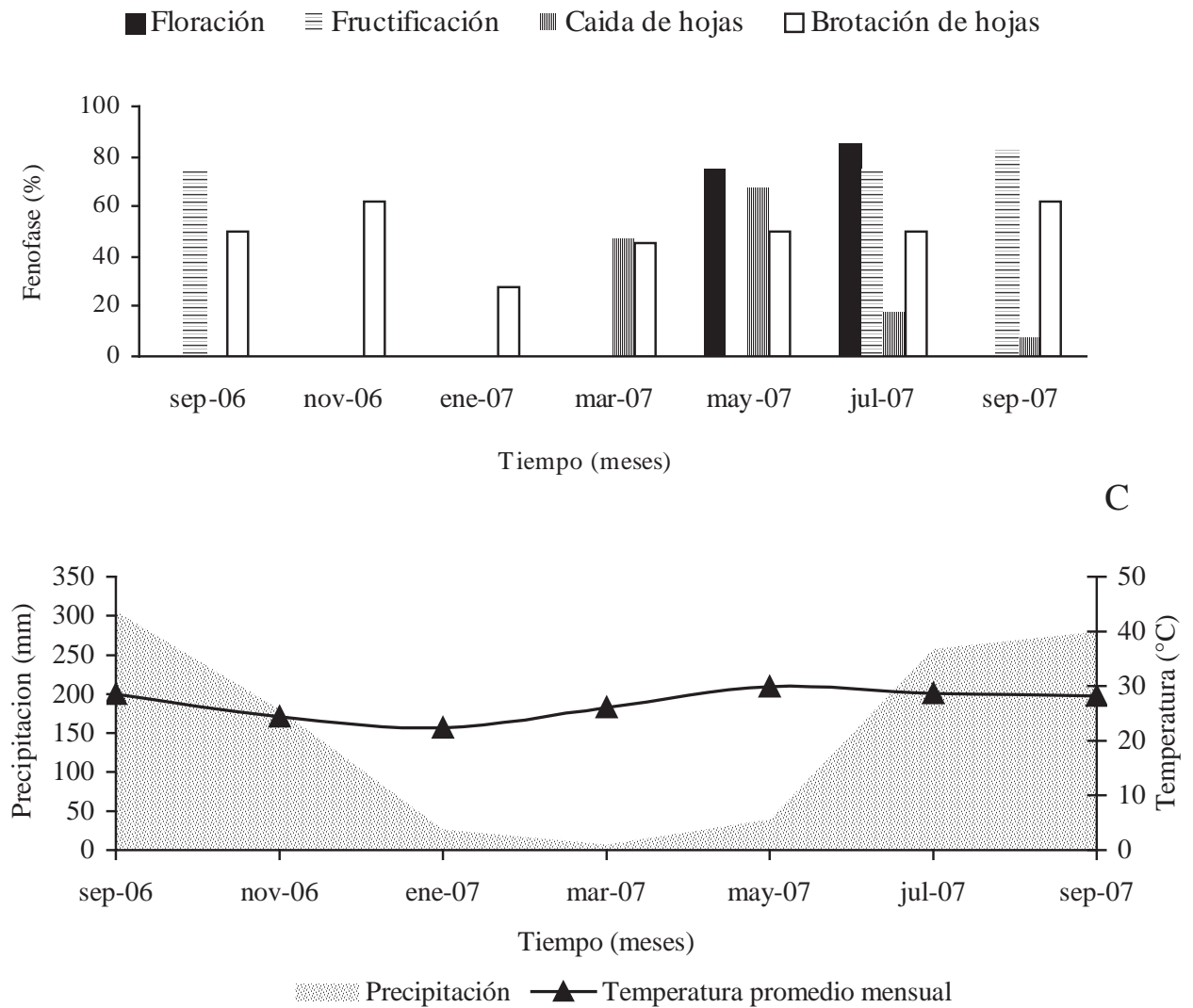

Figura 1. Diagramas fenológicos para Schizolobium parahba (A) y para Vochysia guatemalensis (B) con relación a la precipitación temperatura del aire (C) en San Martín Soolapan, Oaxaca.

\section{Resultados}

En Schizolobium parahyba la floración duró de marzo a mayo y la producción de frutos de julio a septiembre (Cuadro 2, Figura 1A). La caída de hojas ocurrió en la época de mayor temperatura y menor precipitación (Figura 1C), cuando se registraron además los valores más bajos de aparición de hojas nuevas (Cuadro 2). En Vochysia guatemal- ensis, la floración se presentó de mayo a julio y sus frutos de julio a septiembre (Figura 1B). Los valores más altos para caída y brote de hojas se obtuvieron en mayo y noviembre, respectivamente (Cuadro 2).

Se encontraron correlaciones positivas altamente significativas $(P<0.01)$ de la precipitación con la fructificación en ambas especies y de la precipitación con el brote de hojas en Schizolobium parahyba (Cuadro 3). No obstante que tam- 
Cuadro 3. Coeficientes de correlación y su significancia $(* * P<0.01)$ para elementos climáticos con fenofases de Schizolobium parahyba y Vochysia guatemalensis en San Martín Soyolapan, Oaxaca.

\begin{tabular}{|c|c|c|c|c|c|}
\hline Especie & $\begin{array}{l}\text { Variable } \\
\text { climática }\end{array}$ & Floración & $\begin{array}{l}\text { Fructifi- } \\
\text { cación }\end{array}$ & $\begin{array}{c}\text { Caída } \\
\text { de Hojas }\end{array}$ & $\begin{array}{l}\text { Brotación } \\
\text { de Hojas }\end{array}$ \\
\hline \multirow{4}{*}{$\begin{array}{c}\text { Schizolobium } \\
\text { parahyba }\end{array}$} & Precipitación & -0.6913 & $0.8902^{* *}$ & -0.6433 & $0.8778^{* *}$ \\
\hline & Temperatura & 0.3171 & 0.5254 & 0.3562 & 0.0981 \\
\hline & $\begin{array}{c}\text { Temperatura } \\
\text { mínima }\end{array}$ & 0.1265 & 0.6342 & 0.1621 & 0.3022 \\
\hline & $\begin{array}{l}\text { Temperatura } \\
\text { máxima }\end{array}$ & 0.4187 & 0.4418 & 0.4582 & -0.0274 \\
\hline \multirow{4}{*}{$\begin{array}{c}\text { Vochysia } \\
\text { guatemalensis }\end{array}$} & Precipitación & 0.0968 & $0.8959^{* *}$ & -0.6140 & 0.4870 \\
\hline & Temperatura & 0.6682 & 0.5529 & 0.4354 & 0.4360 \\
\hline & $\begin{array}{l}\text { Temperatura } \\
\text { mínima }\end{array}$ & 0.6617 & 0.6585 & 0.2597 & 0.4150 \\
\hline & $\begin{array}{l}\text { Temperatura } \\
\text { máxima }\end{array}$ & 0.6674 & 0.4771 & 0.5295 & 0.4439 \\
\hline
\end{tabular}

bién se obtuvieron altos coeficientes (negativos) de correlación de la precipitación con la floración y caída de hojas en $S$. parahyba, así como de la precipitación con la caída de hojas en Vochysia guatemalensis, estos coeficientes no fueron significativos. De la misma forma, aunque se encon-

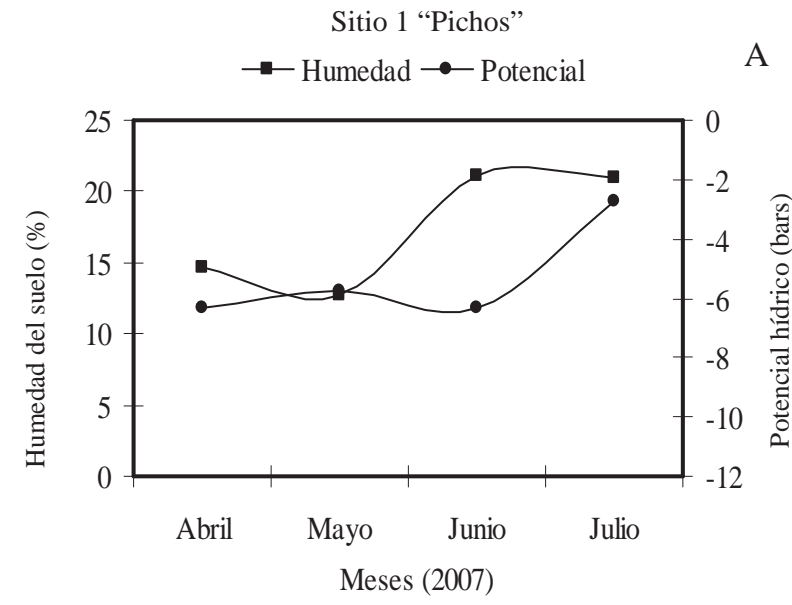

A
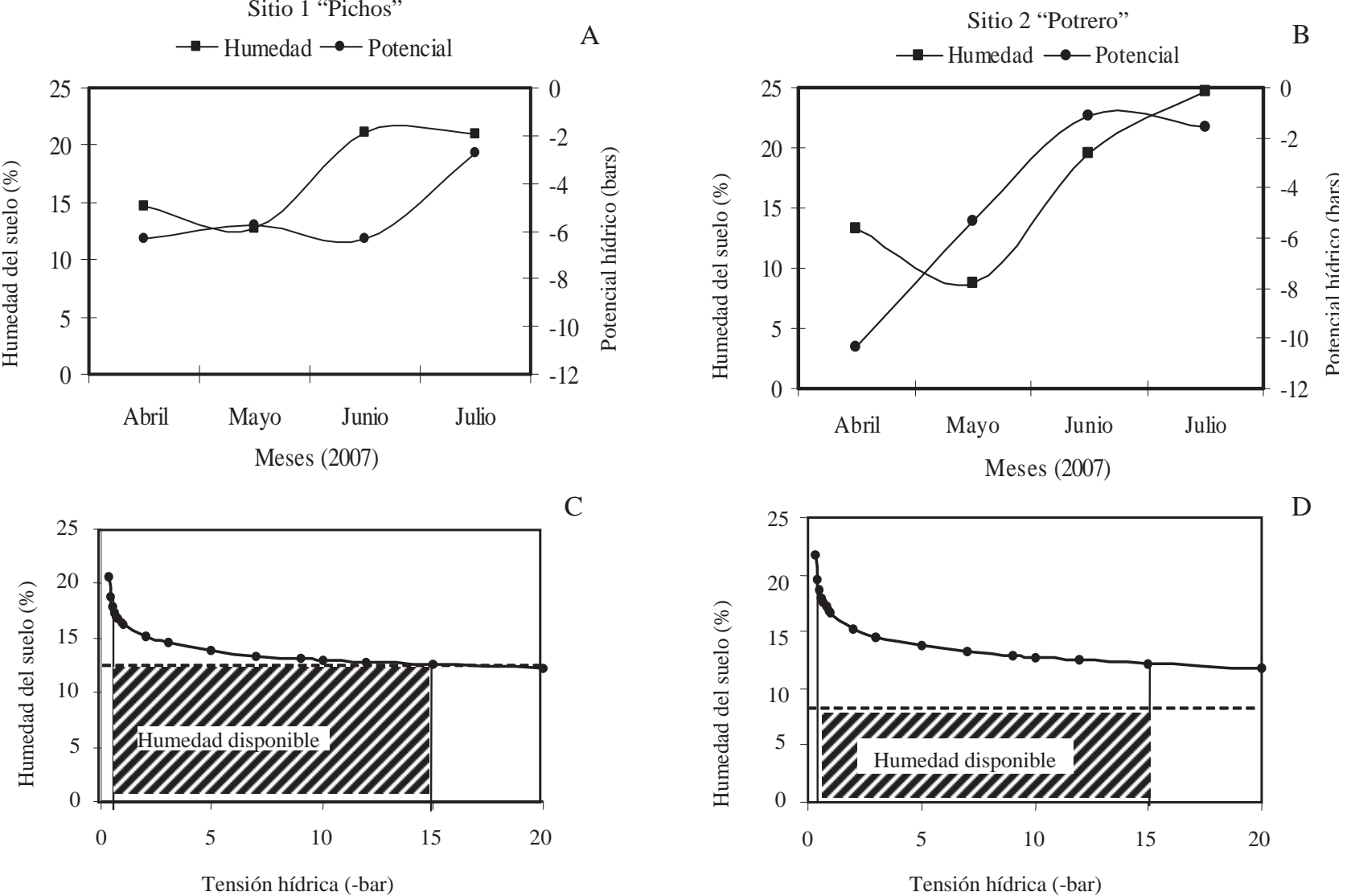

traron correlaciones positivas de la temperatura mínima con la fructificación en ambas especies, así como de las temperaturas promedio, mínima y máxima con la floración de $V$. guatemalensis, estas correlaciones tampoco fueron significativas (Cuadro 3).

Schizolobium parahyba para el sitio "pichos" tuvo valores mínimos de humedad de $13 \%$ en mayo y de $22 \%$ en Junio, sus potenciales fueron el menor de -6 bar en mayo y -2.5 bar en Julio; El sitio "potrero" de $8 \%$ mínimo en el mes de mayo y máximo $25 \%$ en julio. El potencial hídrico en este sitio fue -11 bar en mayo y -1 bar en junio. (Figura 2A, B). Las curvas de retención de humedad muestran que hay diferencias entre sitios por el contenido de agua, principalmente en "potrero"con la menor, en ninguno de los dos casos los árboles se encuentran cercanos al PMP ( $\approx-15$ bar $)$.

El sitio "curva" de Vochysia guatemalensis tuvo en las muestras de suelo una humedad mínima de $19 \%$ en mayo y máxima de $30 \%$ en julio, el potencial hídrico mínimo registrado en mayo de --3.5 bar y máximo en junio de -1.5 bar. En el sitio cafetal la humedad mínima fue de $13 \%$ en mayo y máxima en junio de $28 \%$, los potenciales fueron: el mínimo en mayo con -5.8 bar y máximo en junio con -1.5 bar (Figura 3A, B) fueron respectivamente menores en abril y mayo, cuando la precipitación fue menor (Figura 2E, F).
$\mathrm{C}$

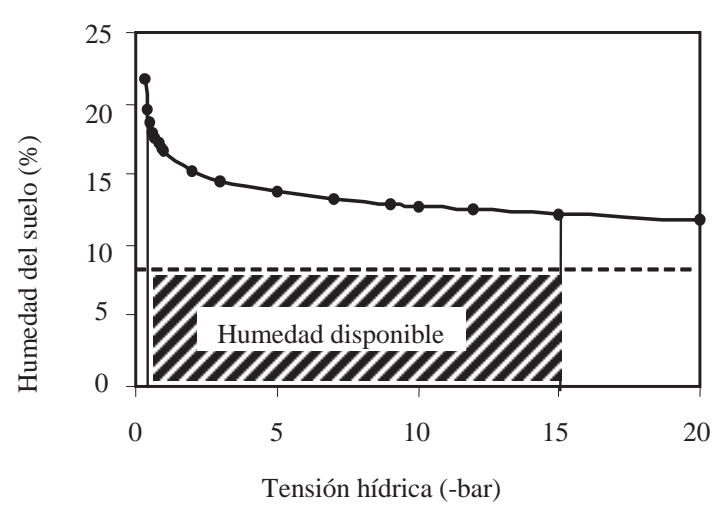$$
0
$$

Figura 2. Humedad del suelo y potencial hídrico de Schizolobium parahba (A y B) y curvas de retención de humedad (C y D). El área achurada indica la disponibilidad de agua en el suelo para los árboles. 


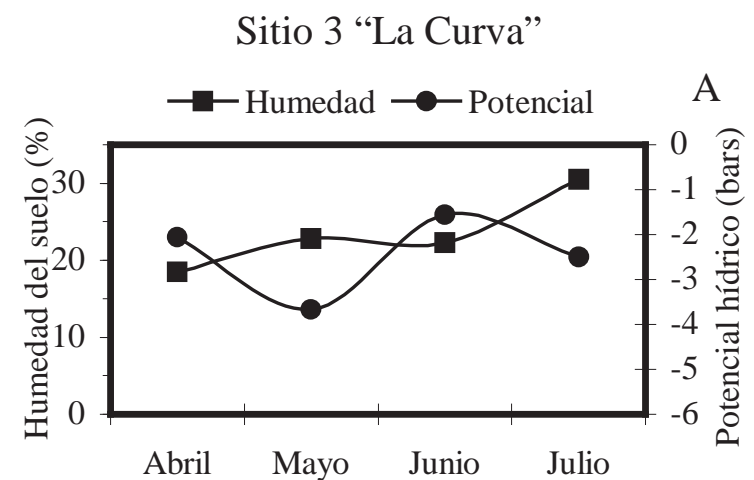

Meses (2007)

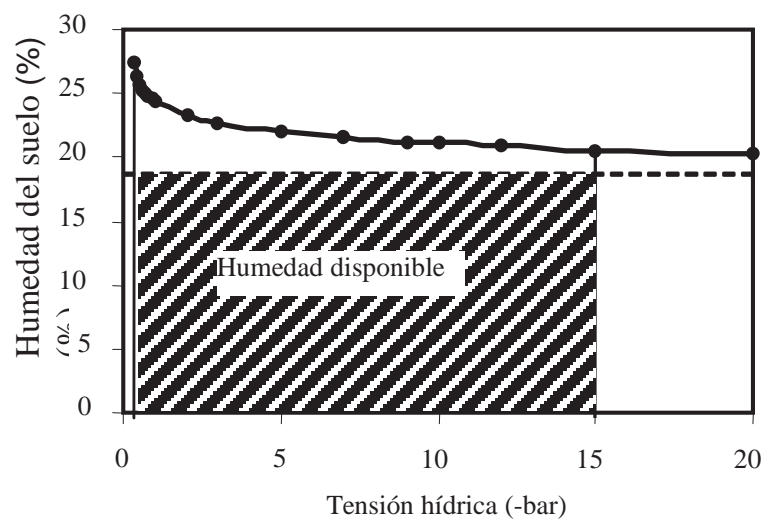

$\mathrm{C}$

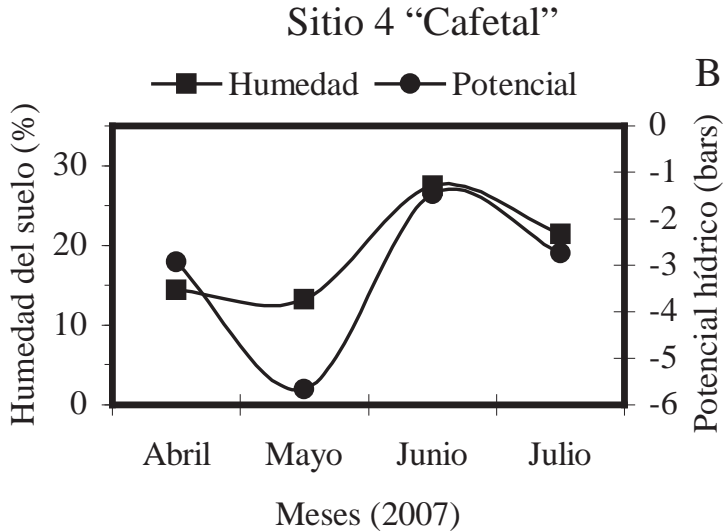

$\mathrm{B}$

$\mathrm{D}$

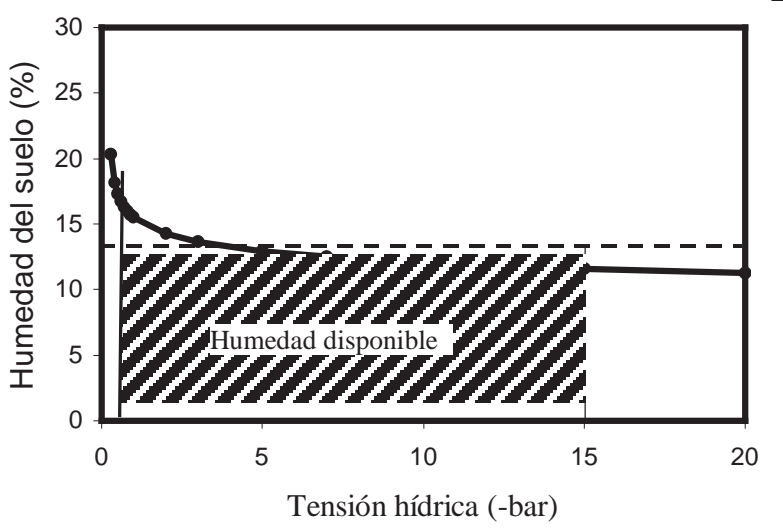

Figura 3. Humedad del suelo y potencial hídrico de Vochysia guatemalensis (A y B) y curvas de retención de humedad (C y D). El área achurada indica la disponibilidad de agua en el suelo para los árboles.

Las curvas de retención de humedad mostraron que los árboles están a CC y lejanos del PMP (Figuras 2C, 3C).

\section{Discusión}

Schizolobium parahyba tiene un patrón estacional de floración en la zona de estudio, coincidiendo con lo reportado por Gómez-Restrepo (2010) en Colombia; Wright (1991) señala que aunque se pueda ver afectada la aparición flores por una sequía, no se pone en riesgo la reproducción del árbol. En el presente estudio, los valores de estrés hídrico medidos se consideran lejanos de un PMP (-15 bar; López, 2005) por lo tanto hay mecanismo que permite que se cumplan las funciones fisiológicas de la producción de flores. Varios autores (Machado-de Carvalho y Dietrich, 1993; Carvalho, 2005; Kamenetsky et al., 2005) mencionan que en estas condiciones las flores aparecen porque hay sustancias de reserva (polisacáridos) que cubren la demanda energética para dicho proceso.

En Vochysia guatemalensis no hay asociación entre la floración y la precipitación, pues se desfasa de los meses con menor lluvia. Este hecho se ha observado en otras selvas perennifolias y se atribuye a mecanismos endógenos llamados "síndromes de dispersión” o aspectos como la radiación solar y duración del día (Morellato et al., 2000; Hamann, 2004; Bendix et al., 2006). Wright y Cornejo (1990) comprobaron experimentalmente que el estrés hídrico en algunas especies arbóreas tropicales no está necesariamente asociado con la periodicidad de la aparición de flores, aún cuando se proporcione agua de forma ininterrumpida durante la época más seca.

Varios trabajos señalan que la fructificación se correlaciona con la precipitación ya que frutos de Leguminosas tuvieron su mayor pico de fructificación durante la época lluviosa (Bendix et al., 2006), al igual que Vochysia guatemalensis (Vilchez et al., 2004) y V. pyramidalis (Silveira et al., 2002) maduraron sus frutos en la época de mayor disponibilidad de agua edáfica y en la planta. Se sabe que la humedad del sitio influye en la cantidad y tamaño de las estructuras reproductivas, ya que es fundamental en el transporte de minerales y carbohidratos para la formación de frutos (Kramer y Boyer, 1995), la mayor humedad encontrada en los sitios de Vochysia se favorece por la textura arcillosa del suelo que posee una mayor cantidad de microporos donde el agua es retenida con más fuerza que en la arena o el limo (Aguilera, 1989; Lamber et al., 2008). 
La caída de hojas en Schizolobium parahyba se relacionó de forma negativa con la precipitación, de acuerdo con Reich y Borchert (1984) y López et al. (2005) la defoliación representa una adaptación contra la pérdida de agua, asegurando la supervivencia de la especie en periodos desfavorables, ya que la superfície de absorción se reduce para mantener el agua de reserva (Taiz y Zeiger, 2002). López et al. (2005) mencionan que al presentarse este déficit hídrico pueden suceder alteraciones anatómicas a veces irreparables, tales como la cavitación de los tejidos de conducción, las cuales pueden amenazar la supervivencia de los árboles. La humedad del suelo en texturas de tipo arenosa-arcillosa se pierde más rápidamente que en aquellos completamente arcillosos, por lo que las plantas deben recurrir a mecanismos para evitar tensiones hídricas cercanas al PMP, como el caso del sitio "potrero" para $S$. parahyba (López, 2005).

Vochysia guatemalensis se defolió con mayor intensidad en mayo y aunque este evento no se relacionó en forma significativa con ninguna de las variables, puede correlacionarse con el aumento de la temperatura y con las horas de brillo solar intenso. En Colombia una especie de Vochysia presenta este comportamiento con el aumento de radiación (Urrego y del Valle, 2001), el cual también se atribuye a la duración del día (Morellato et al., 2000); así como otras especies de árboles en selvas perennifolias de América (Rivera y Borchert, 2001).

Los mínimos porcentajes de brote de hojas en Schizolobium parahyba durante la época de menor precipitación y su aumento en los meses de mayor humedad coinciden con lo reportado por Morellato et al. (2000) para la costa atlántica de Brasil donde existe correlación entre los meses menos húmedos con la caída y mayor humedad con aparición de hojas nuevas. En este sentido, se atribuye al estrés hídrico la afectación del crecimiento celular, lo que repercute en la formación de hojas nuevas y en el crecimiento del árbol (Wright, 1991). En Brasil, se encontró que esta especie sometida a una rehidratación presenta un aumento en la cantidad de aminoácidos y proteinas (Carvalho, 2005). Santiago y Mulkey (2005) señalan para especies de las familias Fabaceae y Vochysiaceae en Panamá, que la disminución estacional de la humedad en el suelo afecta la producción de hojas y se recupera con mayor precipitación.

La fenología estacional para Schizolobium parahyba por sensibilidad a la disminución de agua en el suelo se comprobó en este estudio, Vochysia guatemalensis no presentó un patrón fenológico estacional, pues algunas de sus fenofases se ajustan más a hechos geográficos como su distribución latitudinal y a la duración del fotoperiodo. Sin embargo, se requieren futuros estudios que aborden el uso del agua por las especies de interés (ver por ejemplo: Jackson et al., 1999; Liu et al., 2010) para contribuir a un mejor entendimiento de la fenología y dinámica del agua en selvas húmedas.

\section{Agradecimientos}

El Consejo Nacional de Ciencia y Tecnología de México (CONACYT) proporcionó una beca al primer autor para el desarrollo de estudios de posgrado. El Colegio de Postgraduados a través del Programa Forestal apoyo logística y administrativamente. El Comisariado de Bienes Comunales de Comaltepec, Oaxaca brindó las facilidades necesarias para realizar la investigación. Filemón Manzano y los habitantes de San Martín Soyolapan ayudaron en campo.

\section{Literatura citada}

Aguilera H.N. 1989. Tratado de Edafología de México. Tomo I. Universidad Nacional Autónoma de México, México, D.F.

Baker T.R., Affum-Baffoe K., Burslem D.F.R.P. y Swaine M.D. 2002. Phenological differences in tree water use and the timing of tropical forest inventories: conclusions from patterns of dry season diameter change. Forest Ecology and Management 171:261-274.

Bhat D.M. 1992. Phenology of tree species of tropical moist forest of Uttara Kannada district, Karnataka, India. Journal of Biosciences 17:325-352.

Bawa K.S. 1989. Phenology. En: Bawa K.S., Ashton P.S., Primack R.B., Terborgh J., Mohd-Nor S. Ng F.S.P. y Hadley M. Eds. Reproductive Ecology of Tropical Forest Plants. Research Insights and Management Implications, pp. 11-14, The International Union of Biological Sciences, Paris.

Bendix J., Homeier J., Cueva-Ortiz E., Emck P., Breckle S.W., Richter M. y Beck E. 2006. Seasonality of weather and tree phenology in a tropical evergreen mountain rain forest. International Journal of Biometeorology 50:370-384.

Bollen A. y Donati G. 2005. Phenology of the littoral forest of Sainte Luce, southeastern Madagascar. Biotropica 37:32-43.

Borchert R. 1983. Phenology and control of flowering in tropical trees. Biotropica 15:81-89.

Borchert R. 1999. Climatic periodicity, phenology, and cambium activity in tropical dry forest trees. IAWA Journal 20:239-247.

Brenes-Cambronero L. y D'Stefano J.F. 2002. Comportamiento fenológico del árbol Elaeagia uxpanapensis (Rubiaceae), en un bosque pluvial premontano de Costa Rica. Revista de Biología Tropical 49:989-998.

Carabias-Lillo J. y Guevara-Sada S. 1985. Fenología de una selva tropical húmeda en una comunidad derivada, Los Tuxtlas, Veracruz. En: Gómez-Pompa A. y Del Amo S. Eds. Investigaciones Sobre la Regeneración de Selvas Altas en Veracruz, México, pp. 27-66, Instituto Nacional de Investigación Sobre Recursos Bióticos/Editorial Alhambra Mexicana, México, D.F.

Carvalho C.J.R. 2005. Respostas de plantas de Schizolobium amazonicum (S. parahyba var. amazonicum) e Schizolobium parahyba (Schizolobium parahybum) à deficiência hídrica. Árvore 29:907-914.

Chairul T. y Yamauchi J. 2010. Phenological study on a tropical secondary forest in west Sumatra, Indonesia. Tropics 18:211220.

COSEFORMA [Cooperación en los Sectores Forestal y Maderero]. 2001. Cebo en la Zona Norte de Costa Rica. Cooperación en los Sectores Forestal Maderero. Informe técnico. San José. 
De Teresa A.P. 1999. Población y recursos en la región Chinanteca de Oaxaca. Desacatos 1:121-152.

Díaz M. 2001. Ecología experimental y ecofisiología: Bases para el uso sostenible de los recursos naturales de las zonas áridas neo-tropicales. Interciencia 26:472-478.

Fournier L.A. 1974. Un método cuantitativo para la medición de características fenológicas en árboles. Turrialba 24:422-423.

Fournier L.A. y Charpantier C. 1975. El tamaño de la muestra la frecuencia de las observaciones en el estudio de las características fenológicas de los árboles tropicales. Turrialba 25:45-48.

Fournier L.A. 1976. El dendrofenograma, una representación gráfica del comportamiento fenológico de los árboles. Turrialba 26:96-97.

García E. 1987. Modificaciones al Sistema de Clasificación Climática de Köppen. Universidad Nacional Autónoma de México. México, D.F.

García E.G. y Di Stéfano J. F. 2005. Fenología de árbol Sideroxylon capiri (Sapotaceae) en el bosque seco tropical de Costa Rica. Revista de Biología Tropical 53:5-14.

Gómez-Figueroa P. y Fournier L. A. 1995. Fenología y ecofisiología de dos poblaciones de Tabebuia rosea ("Roble de Sabana") en Costa Rica (Scrophulariales: Bignoniaceae). Revista de Biología Tropical 44:61-70.

Gómez-Restrepo M. A. 2010. Fenología Reproductiva de Especies Forestales Nativas Presentes en la Jurisdicción de CORANTIOQUIA. Un Paso Hacia su Conservación Corporación Autónoma Regional del Centro de Antioquia, Antioquia.

Hamann A. 2004. Flowering and fruiting phenology of a Philippine submontane rain forest: climatic factors as proximate and ultimate causes. Journal of Ecology 92:24-31.

Hartshorn G.S. 1995. Ecological basis for sustainable development in tropical forests. Annual Review of Ecology and Systematics 26:155-175.

Haugaasen T. y Peres C.A. 2005. Tree phenology in adjacent amazonian flooded and unflooded Forests. Biotropica 37:620-630.

Ibarra-Manríquez G., Sánchez-Garfias B. y González-García L. 1991. Fenología de lianas y árboles anemócoros en una selva cálido-húmeda de México. Biotropica 23:242-254.

INEGI [Instituto Nacional de Estadística y Geografía]. 2011. Prontuario de información geográfica municipal de los Estados Unidos Mexicanos ?http://www.inegi.org.mx/sistemas/mexicocifras/datos-geograficos/12/12038.pdf? (consultado 19 de Mayo de 2011).

Jackson P.C., Meinzer F.C., Bustamante M., Goldstein G., Franco A., Rundel P.W., Caldas L., Igler E. y Causin F. 1999. Partitioning of soil water among tree species in a Brazilian Cerrado ecosystem. Tree Physiology 19:717-724.

Kamenetsky R., Peterson R.L., Melville L.H., Machado C.F. y Bewle J.D. 2005. Seasonal adaptations of the tuberous roots of Ranunculus asiaticus to desiccation and resurrection by changes in cell structure and protein content. New Phytologist 166:193-204.

Kramer P.J. y Boyer J.S. 1995. Water Relations of Plants and Soils. Academic Press, Nueva York.

Lambert F. y Marshall A. 1991. Keystone characteristics of birddispersed Ficus in a Malaysian lowland rain forest. The Journal of Ecology 79:793-809.

Lamber H., Chapin F. S. y Pons T.L. 2008. Plant Physiological Ecology. Springer Science, Nueva York.

Liu W., Liu W., Li P., Duan W. y Li H. 2010. Dry season water uptake by two dominant canopy tree species in a tropical seasonal rainforest of Xishuangbanna, SW China. Agricultural and Forest Meteorology 150:380-388.

López G.F. 2005. Ecofisiología de Árboles. Universidad Autónoma Chapingo, Chapingo.

Lopez O.R., Kursar T.A., Cochard H. y Tyree M.T. 2005. Interspecific variation in xylem vulnerability to cavitation among tropical tree and shrub species. Tree Physiology 25:1553-1562.

Machado-de Carvalho M.A. y Dietrich S.M.C. 1993. Variation in fructan content in the underground organs of Vernonia herbacea (Vell.) Rusby at different phenological phases. New Phytologist 123:735-740.

Marques D.S.M.J. 2004. Probabilidad y Estadística para Ciencias Químico-Biológicas. Facultad de Estudios Superiores Zaragoza. Universidad Nacional Autónoma de México, México, D.F.

Miranda F. y Hernández-X. E. 1963. Los tipos de vegetación de México y su clasificación. Boletín de la Sociedad Botánica de México 28:29-179.

Morellato P.C., Talora D.C., Takahashi A., Bencke C., Romera E.C. y. Zipparro V.B. 2000. Phenology of Atlantic rain forest trees: A comparative study. Biotropica 32:811-823.

O'Brien J., Oberbauer S.F., Clark D.B y Clark D.A. 2008. Phenology and stem diameter increment seasonality in a Costa Rican wet tropical forest. Biotropica 40:151-159.

Ochoa G.S., Pérez H.I. y de Jong B.H.J. 2008. Fenología reproductiva de las especies arbóreas del bosque tropical de Tenosique, Tabasco, México. Revista de Biología Tropical 56:657-673.

Reich P.B. y Borchert R. 1984. Water stress and tree phenology in a tropical dry forest in the lowland of Costa Rica. Journal of Ecology 72:61-74.

Ritchie G.A. y Hinckle T.M. 1975. The pressure chamber as an instrument for ecological research. Advances in Ecological Research 9:165-254.

Rivera G. y Borchert R. 2001. Induction of flowering in tropical trees by a 30-min reduction in photoperiod: evidence from field observations and herbarium specimens. Tree Physiology 21:201-212.

Rosales L., Suhartono-Wijoyo F., Dvorak W.S. y Romero J.L. 1999. Parámetros genéticos variación entre procedencias de Schizolobium parahybum (Vell) Blake establecidas en Venezuela. Foresta Veracruzana 1:13-18.

Santiago L. y Mulkey S. 2005. Leaf productivity along a precipitation gradient in lowland Panama: Patterns from leaf to ecosystem. Trees 19:349-356.

Sakai S., Harrison R.D., Momose K., Kuraji K., Nagamasu H., Yasunari T., Chong L. y Nakashizuka T. 2006. Irregular droughts trigger mass flowering in aseasonal tropical forests in Asia. American Journal of Botany 93:1134-1139.

SMN [Servicio Meteorológico Nacional]. 2007. Datos climáticos años 2006-2007 de la estación Tuxtepec, Oaxaca. Departamento de información estadística. Comisión Nacional del Agua. México, D.F.

Silveira L., Funch R. y Maciel G. 2002. Phenology of gallery and montane forest in the Chapada Diamantina, Bahía, Brazil. Biotropica 34:40-50.

Solís S., Lobo J. y Grimaldo M. 2009. Phenology and recruitment of Caryocar costaricense (Caryocaceae), an endemic tree species of southern Central America. Revista de Biología Tropical 57:771-780.

Soto M. y Gama L. 1997. Climas. En: González-Soriano E., Dirzo 
Fenología de SChizolobium parahyba y Vochysia guatemalensis en una selva alta perennifolia de OaXaca, México

R. y Vogt R. Eds. Historia Natural de Los Tuxtlas, pp. 7-23, Universidad Nacional Autónoma de México/Comisión Nacional para el Conocimiento y Uso de la Biodiversidad, México, D.F.

Stata Corp. 1984-2005. STATA. Statistics/Data Analysis.

Taiz L. y Zeiger E. 2002. Plant Physiology. Benjamin Cummings Publ. Co., Redwood City.

Tutin C.E.G. y Fernández M. 1993. Relationship between minimum temperature and fruit production in some tropical forest trees in Gabon. Journal of Tropical Ecology 9:241-248.

Urrego L. y Del Valle J. 2001. Relación fenología-clima de algunas especies de los humedales forestales (guandales) del pacífico sur colombiano. Interciencia 26:150-156.

Van Schaik. C.P. 1986. Phenological changes in a Sumatran rain forest Journal of Tropical Ecology 2:327-347.

Van Schaick C.P., Terborgh W.J. y Wright S.J. 1993. The phenology of tropical forest: Adaptative significance and consequences for primary consumers. Annual Review of Ecology and Systematics 24:353-377.

Vilchez B., Chazdon R. y Redondo A. 2004. Fenología reproductiva de cinco especies forestales del bosque secundario tropical. Kurú Revista Forestal 1:1-10.

Walter H. 1977. Zonas de Vegetación y Clima. Ed. Omega, Barcelona.

Wright S.J. 1991. Seasonal drought and the phenology of shrubs in a tropical moist forest. Ecology 72:1643-1657.

Wright S.J. y Cornejo F.H. 1990. Seasonal drought and leaf fall in a tropical forest. Ecology 7:1165-1175.

Wright S.J. y Van Schaik C.P. 1994. Light and the phenology of tropical trees. American Naturalist 143:192-199.

Zárate R., Amasifuén C. y Flores M. 2006. Floración y fructificación de plantas leñosas en bosques de arena blanca y de suelo arcilloso en la amazonía peruana. Revista Peruana de Biología 13:095-102.

Recibido: 31 de mayo de 2011

Aceptado: 8 de noviembre de 2011 